\title{
WebMCOT web-service for prediction of co-occurred DNA motifs in ChIP-seq data
}

\author{
Aleksey M. Mukhin \\ Shared Access Center Bioinformatics \\ Institute Cytology and Genetics \\ SB RAS \\ Novosibirsk, Russia \\ mukhin@bionet.nsc.ru
}

\author{
Victor G. Levitsky \\ Laboratory of Evolutionary \\ Bioinformatics and Theoretical \\ Genetics \\ Institute Cytology and Genetics \\ SB RAS \\ Novosibirsk, Russia \\ levitsky@bionet.nsc.ru
}

Sergey A. Lashin

Sector for computer analysis and simulations of biological systems Institute Cytology and Genetics SB RAS

Novosibirsk, Russia

lashin@bionet.nsc.ru

\author{
Dmitriy Y. Oschepkov \\ Laboratory of Evolutionary \\ Bioinformatics and Theoretical \\ Genetics \\ Institute Cytology and Genetics \\ SB RAS \\ Novosibirsk, Russia \\ diman@bionet.nsc.ru
}

\begin{abstract}
Regulation of eukaryotic gene expression is controlled by specific regulatory proteins transcription factors. Binding sites of transcription factors are called motifs. Conventionally, genome-wide annotation of motifs performed with chromatin immunoprecipitation followed by massive sequencing (ChIP-seq) approach. The term composite element (CE) implied two closely located and frequently occurred in genomic DNA motifs. CEs contain two overlapping or spacing motifs. Earlier we proposed Motif Co-Occurrence Tool (MCOT) package that is capable of (a) predicting CEs with both overlapping and spacing of motifs in a single ChIP-seq dataset; (b) all predicted CEs were classified by conservation of both participant motifs. This work presents a web interface WebMCOT for MCOT package.
\end{abstract}

Keywords - web-service, transcription factors, site binding, motifs

\section{Motivation and aim}

Chromatin immunoprecipitation followed by massive sequencing (ChIP-seq) is the most popular approach for experimental analysis of gene expression regulation by transcription factors (TFs) through mapping of their potential binding sites (motifs). Since TF binding conventionally is cooperative, the term composite element (CE) denotes the specific combination of two motifs that exhibit qualitatively new properties, thus resulting in a new pattern of gene regulation; the motifs participating in a $\mathrm{CE}$ may be overlapping, or separated by a relatively short spacer [1].

We recently developed the Motif Co-Occurrence Tool (MCOT) package [2] that is capable of (a) predicting CEs with both overlapping and spacing of motifs in a single ChIP-seq dataset; (b) all predicted CEs were classified to conservation of both participant motifs, i.e. CEs with substantial asymmetry between two participating motifs were also predicted. Hence, MCOT approach have additional advantages as compared with the previous tools $[3,4]$.

MCOT is console program working on Windows and Linux operation systems without graphical user interface (GUI). However, SpaMo tool for prediction of CEs with a spacer [3] have web-interface and service for anyone to apply this approach remotely, so users can use only browser.
WebMCOT provides web-service for application of MCOT package.

\section{Materials and methods}

For web-service development, we use different computer technologies. Firstly, we make client-server architecture through REST API interface between client and server. HTTP requests and responses are compiled with JSON schema. In addition, the "backend" consists of two parts: web-server and worker. They can be on one or more host machines connected by Redis broker messenger.

The JavaScript language, the Vue.JS library for creating the user interface and the Material Design interface style form Google, make frontend site. Backend is made by Python language with Flask library for creating REST API interface and Celery library for running background tasks.

The calculation kernel is written on the $\mathrm{C}++$ language. Also R and Python scripts are developed for visualization.

\section{Results}

In this work, we developed web-service WebMCOT for prediction of co-occurred DNA motifs in ChIP-seq data. The web-service is available on the site: https://webmcot.sysbio.cytogen.ru. The help page was implemented (https://webmcot.sysbio.cytogen.ru/help). The screenshot of WebMCOT interface is provided in Fig. 1. Panel A shows the page that allows to enter input data (ChIP-seq peaks and motifs). The rest panels represent the main output data of WebMCOT. In particular, panel $\mathrm{B}$ shows the significance of various structural types of CEs (see the help page for details); panel $\mathrm{C}$ shows the distribution of mutual locations and orientations for predicted CEs; panel D shows the relative abundances of various $\mathrm{CE}$ types with different ratios of conservation of two participating motifs.

\section{ACKNOWLEDGMENT}

The work is partially supported by RFBR, project no. 1829-13040 "Meta-analysis of genome-wide data based on discovery and annotation of various structural-functional transcription factor binding sites participating in composite elements" and by Budget Project No. 0259-2019-0008. 


\section{REFERENCES}

[1] Kel O.V. et al. (1995) A compilation of composite regulatory elements affecting gene transcription in vertebrates, Nucleic Acids Res., 23(20):4097-4103.

[2] Levitsky V. et al. (2019) A single ChIP-seq dataset is sufficient for comprehensive analysis of motifs co-occurrence with MCOT package, Nucleic Acids Res., 47(21):e139.

[3] Whitington $T$. et al. Inferring transcription factor complexes from ChIP-seq data. // 2011, Nucleic Acids Res., 39(15):e98..

[4] Jankowski A. et al. (2014) TACO: a general-purpose tool for predicting cell-type-specific transcription factor dimers, BMC Genomics, 15:208.

[5] Heinz S. et al. (2010) Simple combinations of lineage-determining transcription factors prime cis-regulatory elements required for macrophage and B cell identities. Mol Cell., 38(4):576-589.
[6] Kulakovskiy I.V. et al. (2018) HOCOMOCO: expansion and enhancement of the collection of transcription factor binding sites models. Nucleic Acids Res., 46(D1), D252-D259.

[7] Ridinger-Saison M. et al. (2012) Spi-1/PU.1 activates transcription through clustered DNA occupancy in erythroleukemia. Nucleic Acids Res., 40(18):8927-8941.

[8] Pang S.H. et al. (2016) PU.1 cooperates with IRF4 and IRF8 to suppress pre-B-cell leukemia. Leukemia. 30(6):1375-1387.

\section{$\equiv$ WebMCOT Application}

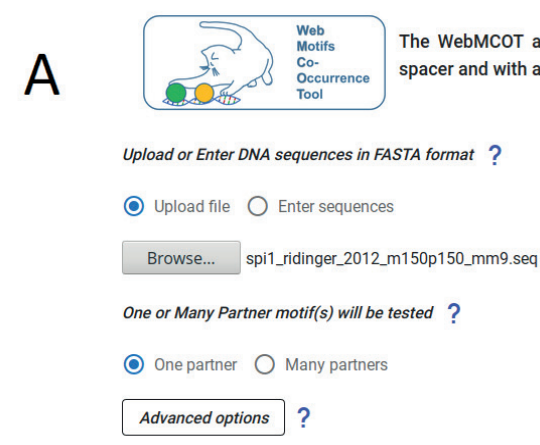

\section{discovers motifs co-occurrence with a}

overlap in a single ChIP-seq dataset

Upload or Enter Anchor motif ?

○ Upload file $\bigcirc$ Enter motif $\bigcirc$ choose motif

Browse... spi1.motif

Upload or Enter Partner motif ?

Select organism to set thresholds of recoonition models

Mouse

(1) Upload file $\bigcirc$ Enter motif $\bigcirc$ choose motif

Browse... in irf.motif

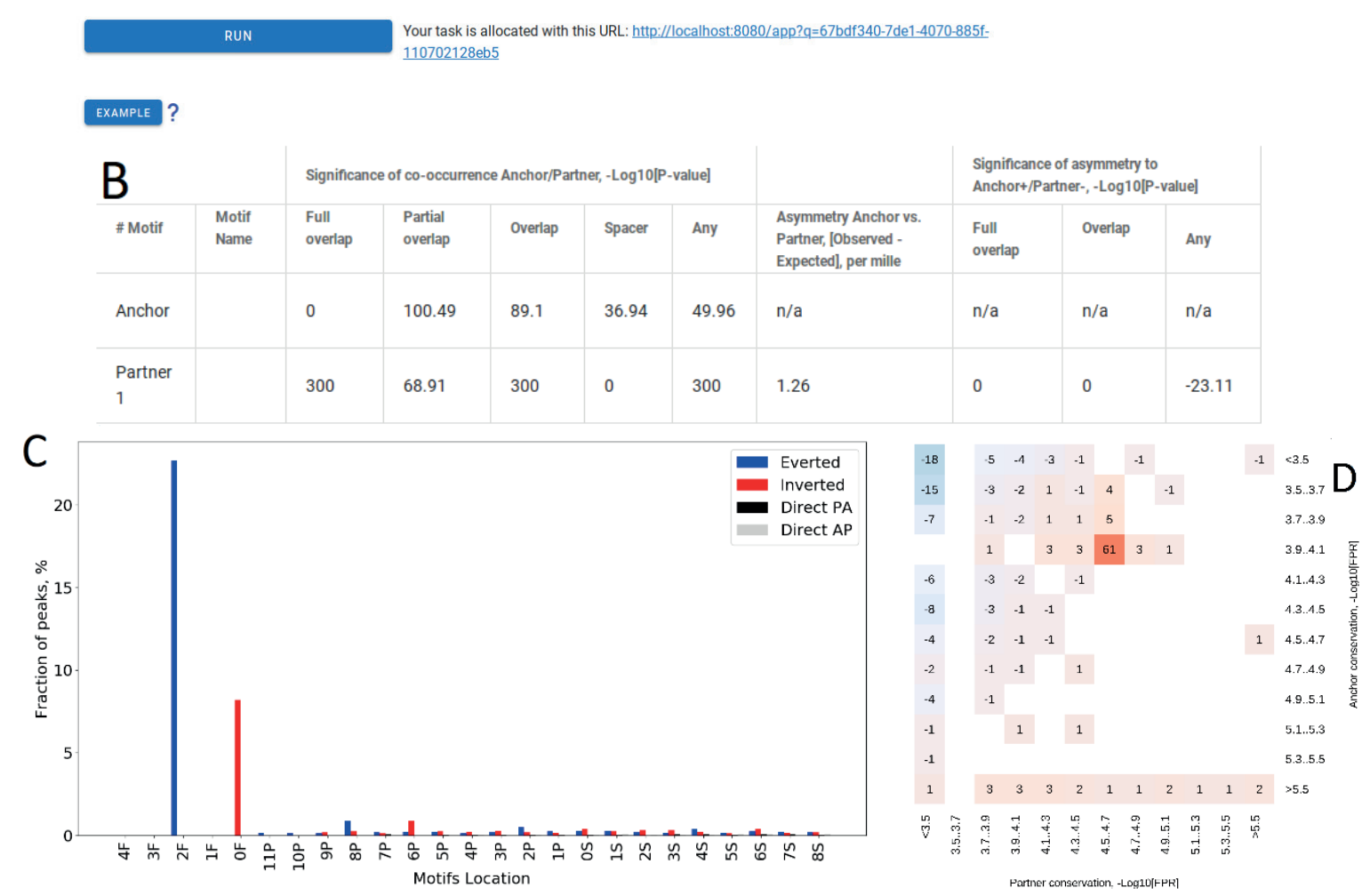

Fig. 1. WebMCOT interface for applying MCOT pipeline. A. The application page for the input data specification (nucleotide sequences of peaks and motifs). B. The summary for the significances of various structural types of predicted CRs. C. Histogram of frequencies of various structural types of CEs according to mutual locations and orientation of motifs. D. The relative abundances of various types of CEs with different ratios of conservation of two motifs. Axes X/Y show the conservation of two motifs, i.e. $-\log _{10}(\mathrm{FPR})$ value (here FPR denotes the false positive rate that respects to predicted hits). The color shows the difference between observed (peaks) and expected (permuted sequences) relative abundance of CEs with specific conservation levels. The Anchor SPI1 and Partner IRF8 motifs were derived from Homer de novo motif search [5] and Hocomoco database [6]. ChiP-Seq peaks were taken from [7]. The previous study [8] have shown that SPI1 and IRF8 cooperate to regulate early B cell development 